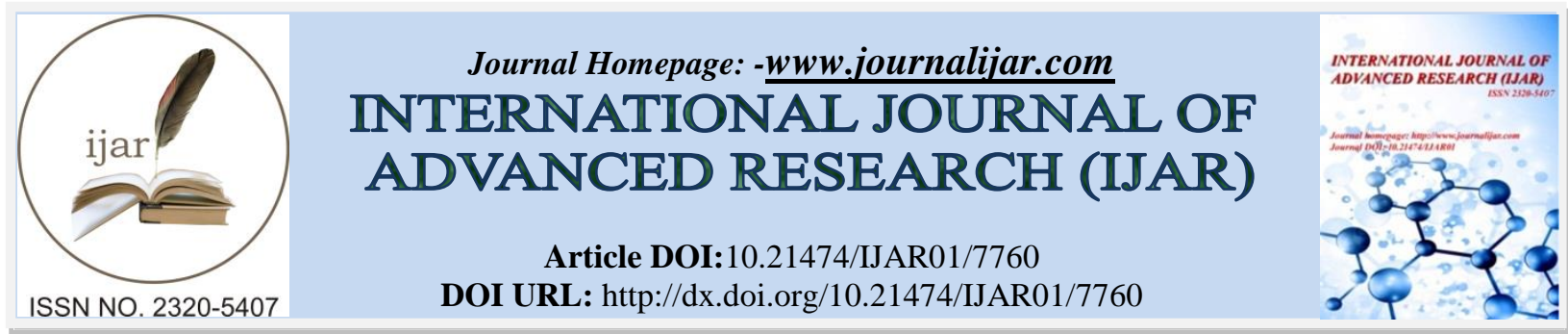

RESEARCH ARTICLE

\title{
RADIX VISTA: A CASE SERIES.
}

Renu Batra ${ }^{1}$, Riddhi Hirani ${ }^{2}$, Kesha Vasavada ${ }^{3}$ and Sonali Kapoor ${ }^{4}$.

1. Reader, Department of Conservative Dentistry and Endodontics, Manubhai Patel Dental College \& Oral Research institute, Vadodara.

2. $3^{\text {rd }}$ Year Post graduate, Department of Conservative Dentistry and Endodontics, Manubhai Patel Dental College $\&$ Oral Research institute, Vadodara.

3. $2^{\text {rd }}$ Year Post graduate ,Department of Conservative Dentistry and Endodontics, Manubhai Patel Dental College \& Oral Research institute, Vadodara.

4. Dean, HOD, Department of Conservative Dentistry and Endodontics, Manubhai Patel Dental College \& Oral Research institute, Vadodara.

\section{Manuscript Info}

Manuscript History

Received: August 2018

Final Accepted: September 2018

Published: September 2018

Keywords:-

Permanent mandibular first molar;Radixentomolaris(RE), radix paramolaris(RP), middle distal canal

\section{Abstract}

With the increasing number of reports of aberrant root canal morphology, the clinician needs to be aware of the variable anatomy.Normally the permanent mandibular first molar has two roots, mesial and distal, but mandibular molars may have an additional root located either buccally (radix paramolaris) or lingually (radix entomolaris). Understanding of the presence of an additional root and its root canal anatomy is essential for successful treatment outcome. Various case reports have been published with the finding of middle mesial canal in mandibular first molar, however finding of three distal canals in distal roots of mandibular first molar is rare. These case series focus mainly on the diagnosis and management of variable root canal anatomy.

Copy Right, IJAR, 2018,. All rights reserved.

\section{Introduction:-}

A complete knowledge and potential variations in the dental anatomy from normal are essential to gain success in endodontic therapy.(1) Root canal anatomy is highly complex and unpredictable. Missed canals and the failure to remove all the microorganisms and pulp remnants from the root canal system are probably the main reasons for persistent infection around endodontically- treated molars.(2).

The first mandibular permanent molar is the earliest permanent posterior tooth to erupt. It is responsible for development of occlusion and important for physiologic functions. Commonly, it is the one most frequently in need of endodontic treatment

Most mandibular first molars have two roots located mesially and distally and three root canals (Barker et al. 1974, Vertucci 1984), but variations in the number of roots and in canal morphology are not uncommon (Skidmore \&Bjorndal 1971).(3) 
The major variant is the occurrence of an additional third root, a supernumerary root typically found lingually. This was first reported by Carabelli (1884) and was termed radix entomolaris (RE) (Bolk 1915)(3).This extra root is typically smaller than the distobuccal root and is usually curved.(4)

Radiographic diagnosis plays a pivotal role in successful endodontic treatment of tooth from all the root canals. So, radiographs taken at different angulations give information about extra canals or roots. Radix entomolaris has an occurrence of less than 5\% in the Indian population.(5)

De Moor et al classified RE into three types. (4,6)

Type I: a straight root or root canal,

Type II: an initially curved entrance which continues as a straight root or root canal.

Type III: an initial curve in the coronal third of the root canal and a second curve beginning in the middle and continuing to the apical third

The aim of this study was to discuss a clinical approach for identification, detection, and endodontic management of the radix entomolaris (RE), radix paramolaris (RP) \& three distal canal in the mandibular first molar.

\section{Case 1:-}

A 40 year old female patient presented with chief complaint of pain in lower right back tooth region of the jaw for the past one month. Clinical examination revealed deep proximal caries in mandibular right first molar (irt 46). The tooth was very sensitive to percussion. On radiographic examination irt 46, apart from deep proximal caries, indistinct periapical radiolucency was seen around the distal roots. Further, the presence of an additional distalroot outline was noticed on the radiograph [Figure 1(a)]. The patient was suggested to undergo root canal treatment. Following the initiation of root canal treatment under rubber dam isolation, The pulp chamber was deroofed with endo access burs, the close inspection of the pulp chamber revealed the presence of two mesial and two distal canal orifices [Figure 1(b)]. The presence of all the orifices were confirmed using an endodontic explorer [DG16]. In the subsequent visits, canals were explored and negotiated using \#08 and \#10 size K-files [DentsplyMaillefer, Ballaigues, Switzerland]. The working length of the canals was determined electronically using an apex locator [J Morita Root Zx mini Apex Locator] and confirmed radiographically [Figure 1(c)].. Canals were cleaned and shaped using rotary Ni-Ti files [ProTaper, Dentsply-Maillefer] and crown-down technique. Canals were irrigated using $2.5 \%$ sodium hypochlorite solution and flushed with 17\% EDTA solution to remove smear layer followed by $2 \%$ chlorhexidine irrigation. Canal disinfection was carried out using calcium hydroxide [Calcicur, VOCO, Germany]. In this patient, the finding of a separate disto-lingual canal orifice and radiographic outline of the roots in the subsequent radiographs indicated the presence of anRE.In the follow up visits, when the patient was found asymptomatic, gutta-percha master cones [ProTaper, DentsplyMaillefer] were selected [Figure 1(d)]. Obturation was carried out with master cones and AH26 sealer [De Trey Dentsply, Konstanz, Germany] [Figure 1(d) and 1(e)]. The access opening was restored with composite [Figure 1(f)]

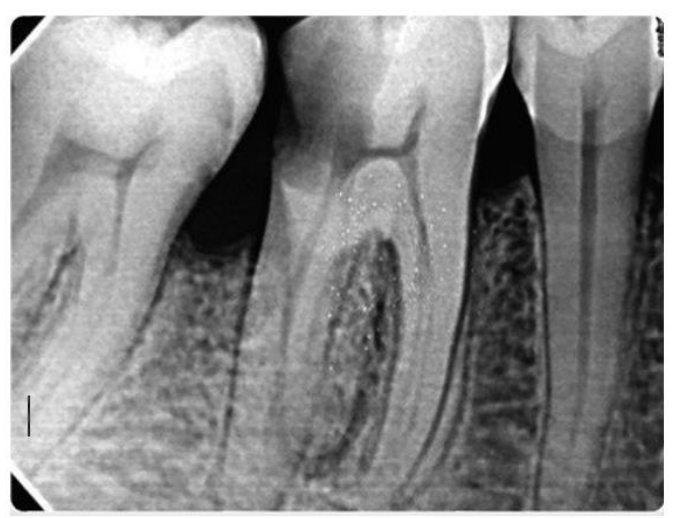

$$
\begin{aligned}
& \text { Figure 1(a)-Pre operative } \\
& \text { Radiograph (the presence of an } \\
& \text { additional distal root outline) }
\end{aligned}
$$

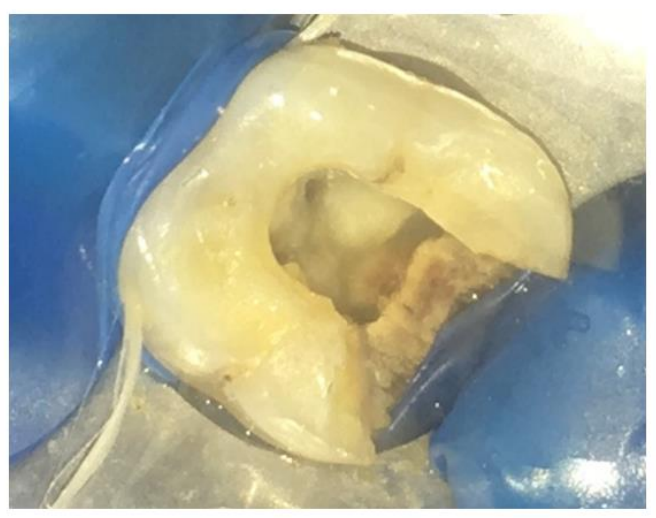

Figure 1(b)- Access Opening (pulp chamber revealed the presence of two mesial and two distal canal orifices) 


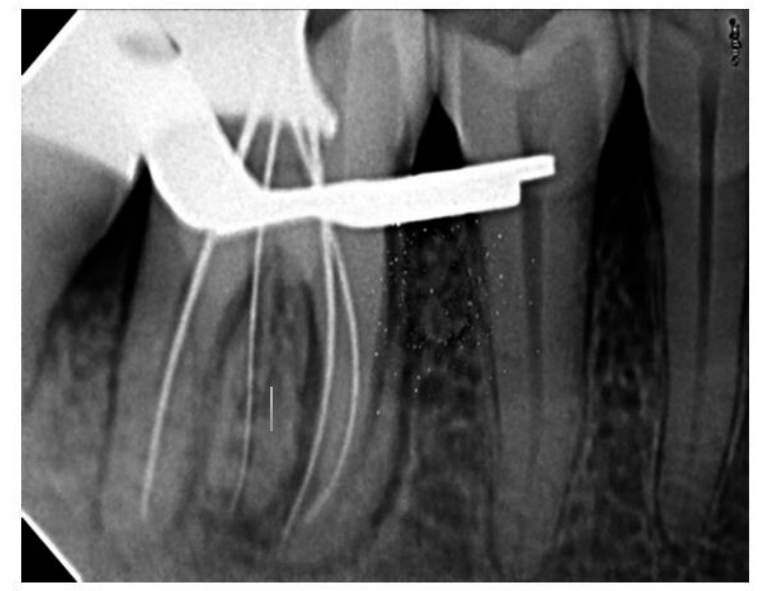

Figure 1(c)- Working length determination

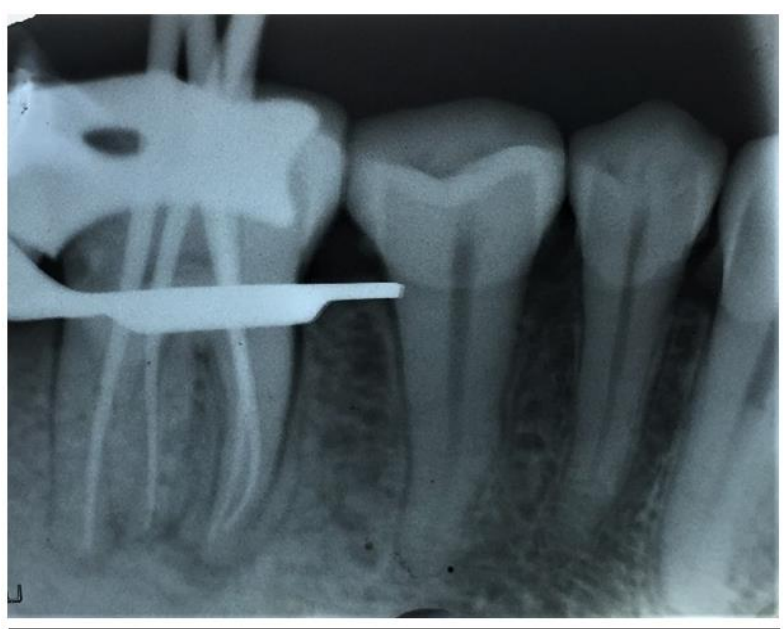

Figure 1(d)- Master cone IOPA

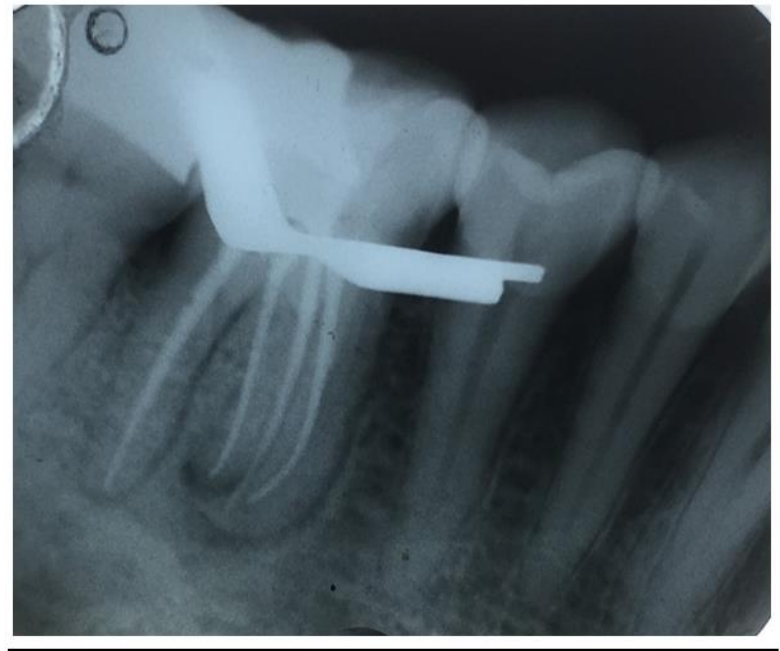

Figure 1(e)- Obturation

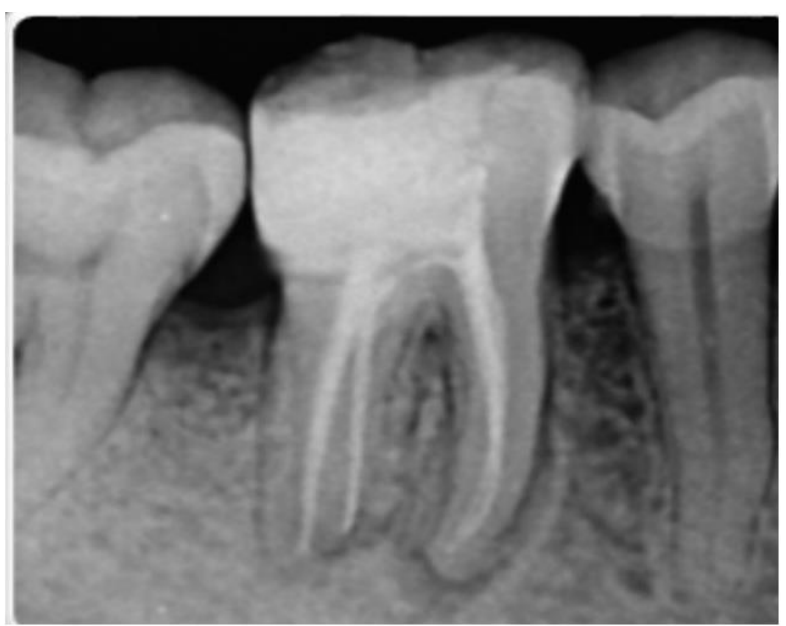

Figure 1(f)- Post obturation

\section{Case 2:-}

A 22 -year-old male patient was presented with dull aching pain while chewing food on mandibular left first molar (tooth 36). Clinical examination revealed deep proximal caries in mandibular left first molar (irt 36). On radiographic examination irt 36, apart from deep proximal caries, indistinct periapical radiolucency was seen around the both roots (mesial \& Distal) . It also showed a double mesial root outline indicating the presence of a radix paramolaris (RP) [Figure 2a]. Root canal treatment was started under rubber dam isolation. After achieving adequate anesthesia, the pulp chamber was deroofed with endo access burs, the close inspection of the pulp chamber revealed the presence of two mesial and one distal canal orifices [Figure 2(b)]. The endodontic procedure was carried out similar to case 1 . Following canal negotiation, working length was determined [Figure 2(c)] and canals were cleaned and shaped in a crown-down manner using rotary $\mathrm{Ni}-\mathrm{Ti}$ files and $2.5 \%$ sodium hypochlorite and $17 \%$ EDTA solutions as irrigants during instrumentation. Canals were disinfected using metapex as an intracanal medicament for 
period of 1 month[Figure 2(d)]. Subsequently, canals were obturated with gutta-percha point master cones and AH26 sealer [Figure 2(e) and 2(f)]. The access opening was restored with composite [Figure 2(g)]

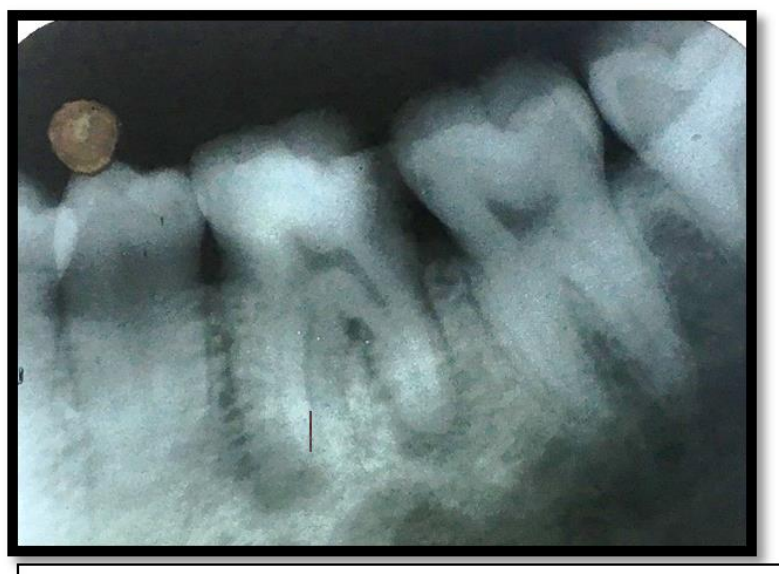

Figure 2(a)-Pre operative Radiograph (the presence of an additional distal root outline)

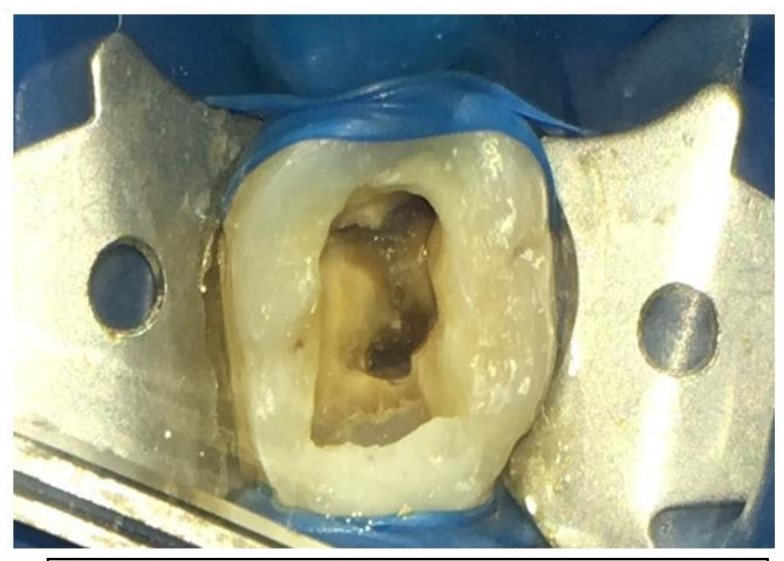

Figure 2(b)- Access Opening (pulp chamber revealed the presence of two mesial and one distal canal orifices)

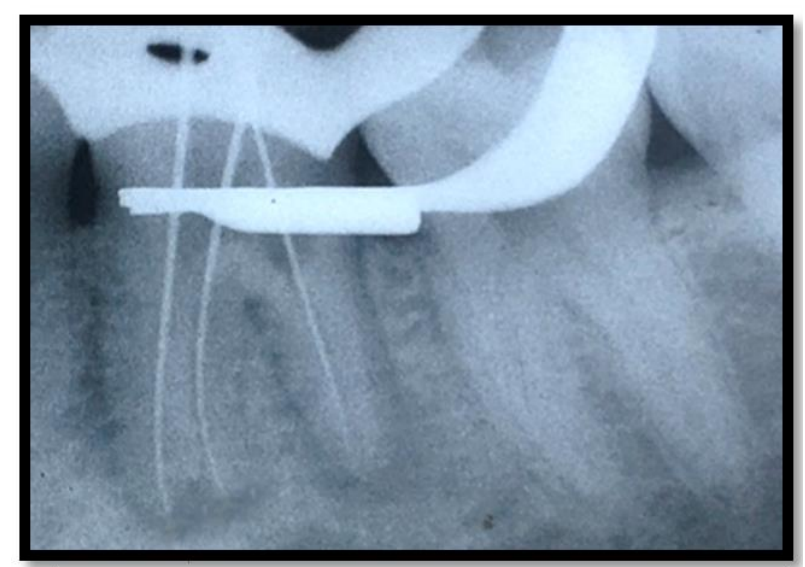

Figure 2(c)- Working length determination

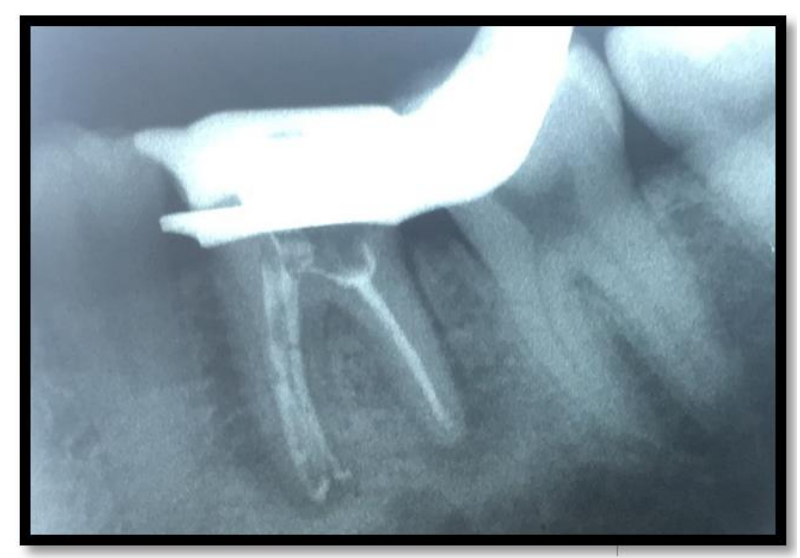

Figure 2(d)- Metapex placement 

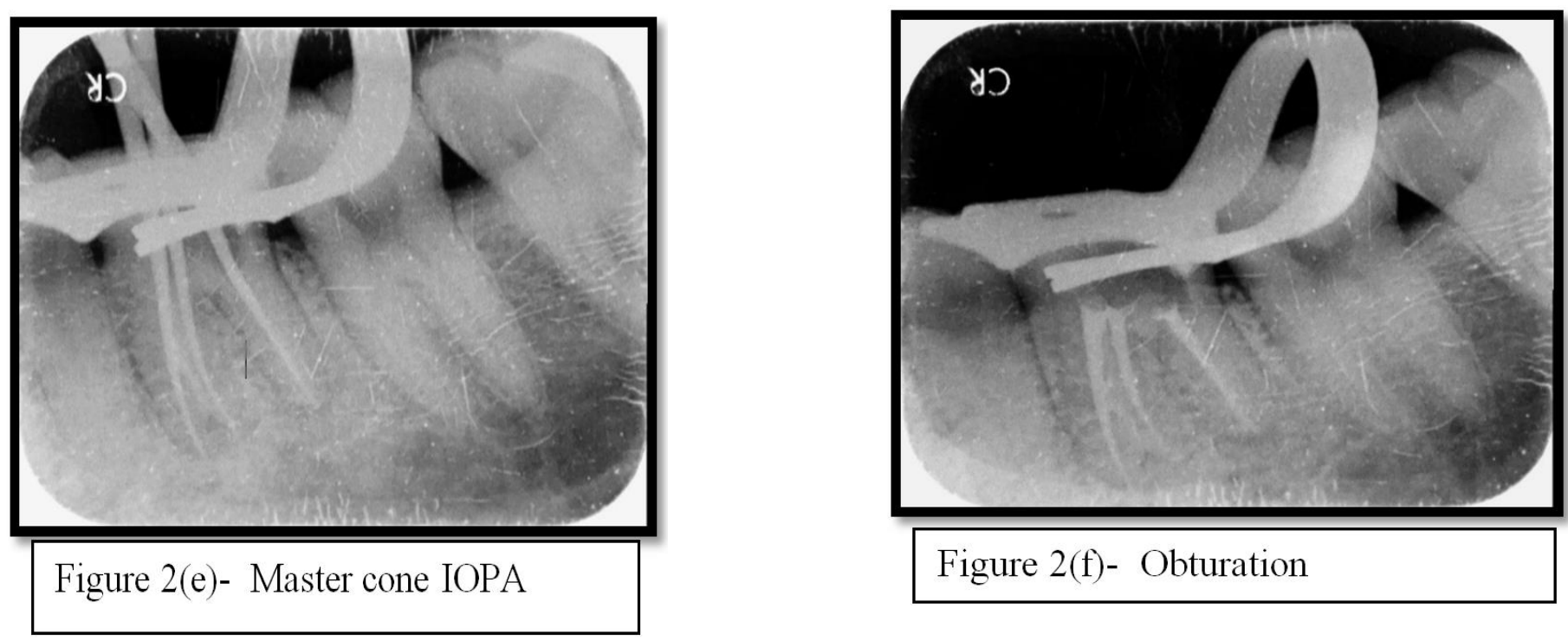

Figure 2(f)- Obturation

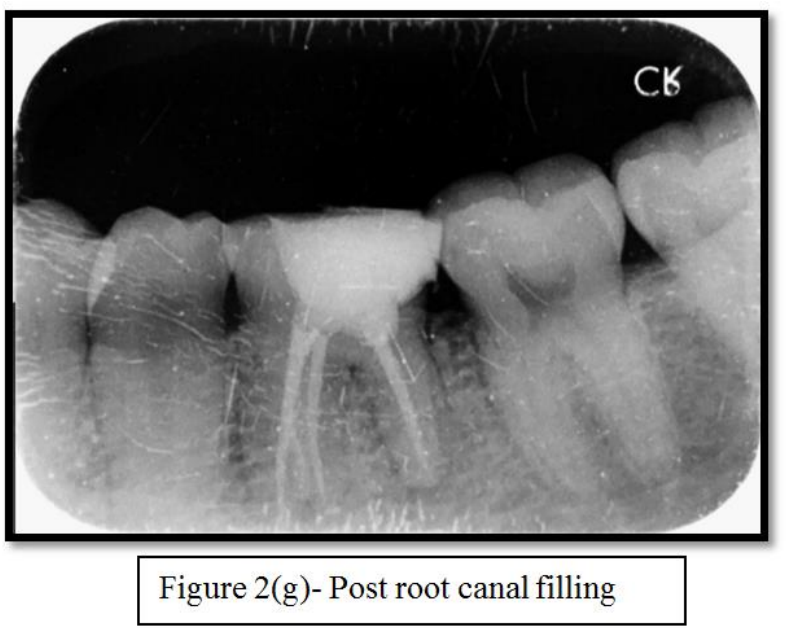

Case 3:-

A male patient, aged 33 years old, reported with a chief complaint of pain in the lower right back tooth region since 1 month.On clinical examination, there was a deep Class II distoocclusal caries in relation to tooth (irt 46) which was tender on percussion. Thermal and electric pulp test elicited a negative response.on radiographic examination irt46, apart from deep proximal caries, indistinct periapical radiolucency was seen around the roots. Further, the presence of an additional distalroot outline was noticed on the radiograph [Figure 3(a)]. The patient was suggested to undergo root canal treatment. Following the initiation of root canal treatment under rubber dam isolation, After endodontic access preparation, inspection of the pulp chamber floor revealed two mesial and two distal canals. On careful examination with magnification loupes (Seiler) one additional distal canal orifice (distobuccal 2) was identified in the groove, present between the distobuccal and the distolingual canal orifices. The presence of all the orifices were confirmed using an endodontic explorer [DG16].and subsequently an additional canal was negotiated using \#08 and \#10 size K-files [DentsplyMaillefer, Ballaigues, Switzerland] [Figure 3(b)]. Three canals (distobuccal 1, distobuccal 2 and distolingual) canal were negotiated. The working length of the canals was determined electronically using an apex locator [J Morita Root Zx mini Apex Locator] and confirmed radiographically.Working length radiograph revealed that distobuccal 1 and distobuccal 2 were merging near the apex and distolingual canal was present independently [Figure 3(c)]. All the Canals were instrumented with Protaper rotary system till size F3 (Dentsply, Maillefer) and crown-down technique. Canals were irrigated using $2.5 \%$ sodium hypochlorite solution and flushed with 17\% EDTA solution to remove smear layer followed by $2 \%$ chlorhexidine irrigation. Canal disinfection was carried out using calcium hydroxide [Calcicur, VOCO, Germany]. In the follow up visits, when the patient was found asymptomatic, gutta-percha master cones [ProTaper, DentsplyMaillefer] were selected [Figure 
3(d)]. Obturation was carried out with master cones and AH26 sealer [De Trey Dentsply, Konstanz, Germany] [Fig.3(d), Fig.3(e)]. The access opening was restored with composite [Figure 3(f)]

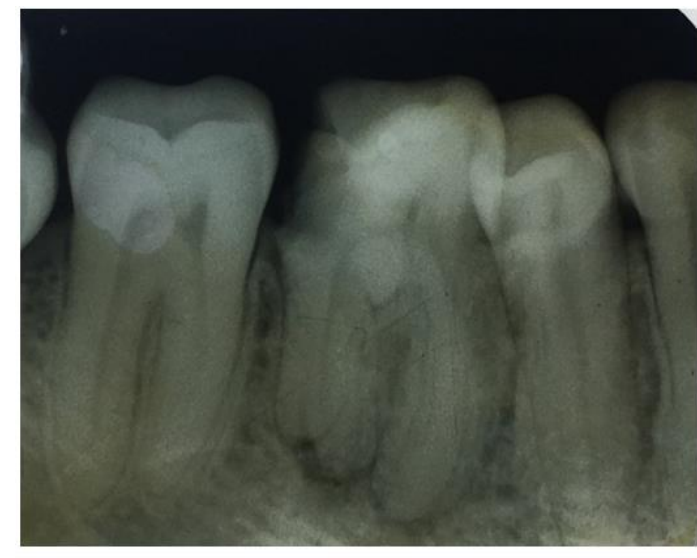

Figure 3(a)-Pre operative Radiograph (the presence of an additional distal root outline)

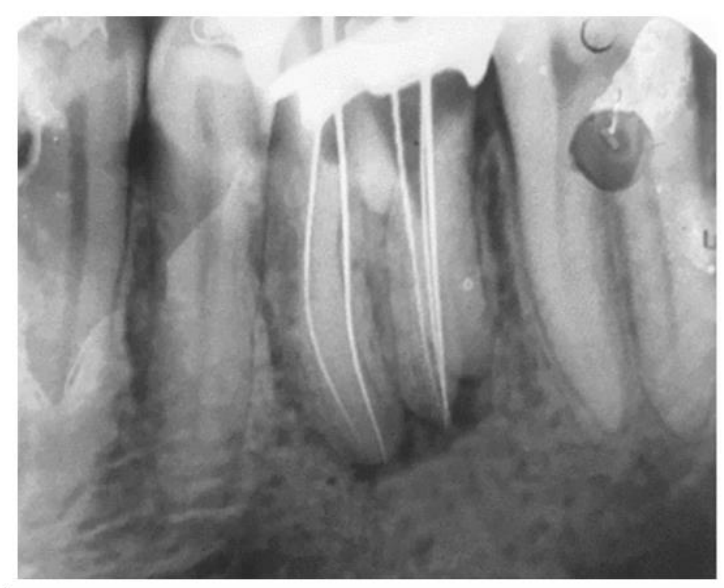

Figure 3(c)- Working length determination

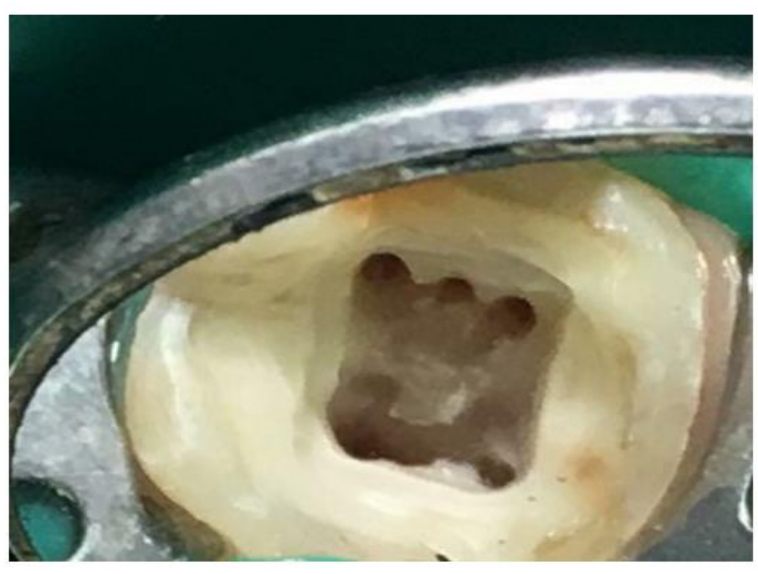

Figure 3(b)- Access Opening (pulp chamber revealed the presence of two mesial and three distal canal orifices)

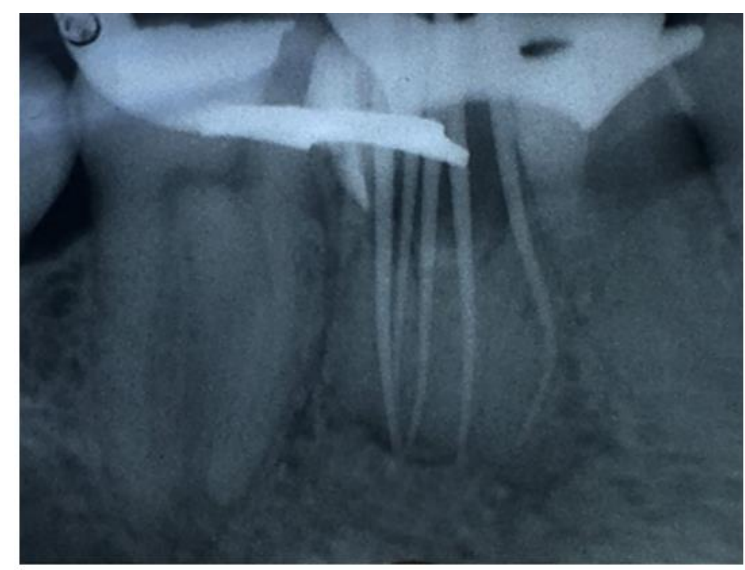

Figure 3(d)- Master cone IOPA

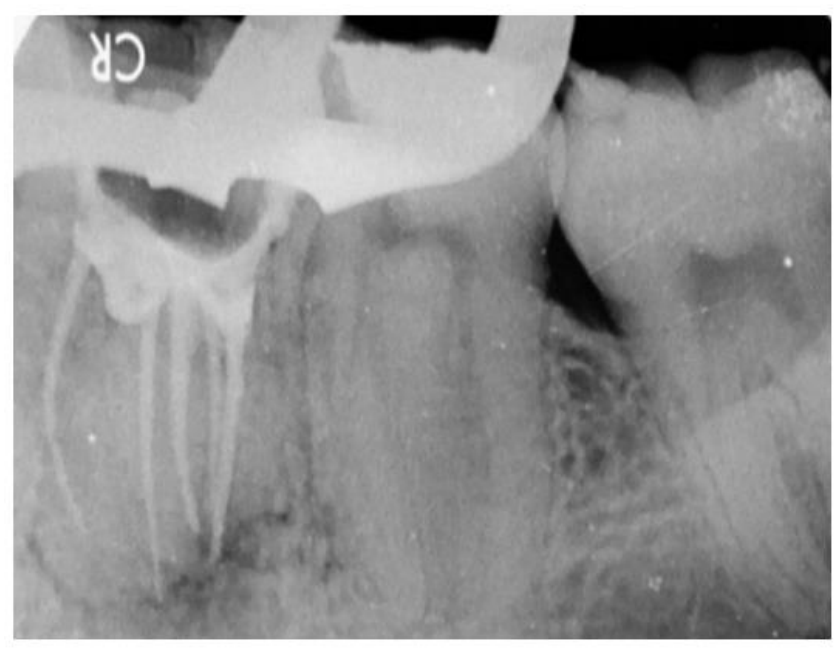




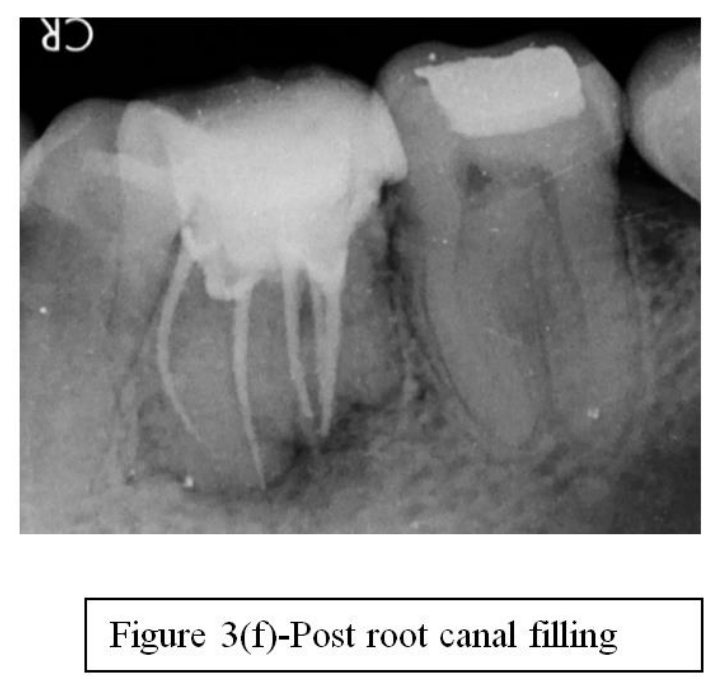

\section{Discussion:-}

Anatomic variations in permanent mandibular first molars are documented in literature. The mandibular first molars usually have two roots, one mesial and one distal, with usual root canal distribution as two canals in the mesial root and one or two canals in the distal root.[1] The presence of a middle mesial canal with incidence of 1-15\% is major variant of root canal system of mandibular first molar. Other common variant is three canals in the distal root with an incidence of $1.7 \%$ in the Indian population; $0.2 \%$ in Senegalese population; $1.7 \%$ in Turkish population; $0.7 \%$ in Burmese population and 3\% in Sudanese population. An extra distobuccal or distolingual root is also encountered in some cases.

The etiology and morphogenesis of radix entomolaris is still not fully understood; it can be attributed to the external factors during tooth formation or atavicgene or polygenic system(5). Carlsen\&Alexandersen (1990) classified radix entomolaris (RE) into four typesdepeding upon the location of its cervical part (7):

1. Type A: the RE is located lingually to the distal root complex which has two cone-shaped macrostructures.

2. Type B: the RE is located lingually to the distal root complex which has one cone-shaped macrostructures.

3. Type $\mathrm{C}$ : the RE is located lingually to the mesial root complex.

4. Type AC: the RE is located lingually between the mesial and distal root complexes.

De Moor et al. (2004) classified radix entomolaris based on the curvature of the root or root canal (4):

1. Type 1: a straight root or root canal.

2. Type 2: a curved coronal third which becomes straighter in the middle and apical third.

3. Type 3: an initial curve in the coronal third with a second buccally oriented curve which begins in the middle or apical third.

Song JS et al. (2010) further added two more newly defined variants of RE (8):

1. Small type: length shorter than half of the length of the distobuccal root.

2. Conical type: smaller than the small type and having no root canal within it.

Morphology: The radix entomolaris is located distolingually ranging from short, conical extension to normal mature root length with its coronal third partially or completely fixed to distal root. Generally, the radix entomolaris is smaller than mesio- and distobuccal roots and may contain pulpal tissue [22]. Externally, the distal furcation is slightly lower $(1 \mathrm{~mm}$.) than the furcation between mesial and distal roots $\mathbf{( 9 )}$

Clinically, tooth with additional distolingual root may present a more bulbous crown outline, an additional cusp, a prominent distolingual lobe or cervical prominence. These features can indicate the presence of additional root. Radiographically, third root is visible in $90 \%$ of cases (10). Occasionally it may be missed because of its slender dimension or overlapping with distal root. Radiographs should be carefully inspected to reveal the presence of hidden radix entomolaris which might present as unclear outline of distal root or root canal. Additional radiographs 
taken from different horizontal projections, 20 degree from mesial and 20 degree from distal reveals the basic information about the anatomy of additional third root $(\mathbf{1 1 , 1 2 )}$.

\section{Radix paramolaris (additional root located buccally):-}

Radix paramolaris is rare entity and occurs less frequently than radix entomolaris (12). Visser reported the prevalence of radix paramolaris to be $0 \%, 0.5 \%$, and $2 \%$ for mandibular first molar, second molars and third molars respectively (1).

Carlsen and Alexandersen (1991) classified radix paramolaris (RP) into two different types (13)

1. Type A: cervical part is located on the mesial root complex.

2. Type B: cervical part is located centrally, between the mesial and distal root complexes.

The radix paramolaris (RP) presents as short conical exension or a mature root, separate or fused, located mesiobuccaly. An additional root is almost always associated with an increased number of cusps and increased number of root canals but vice-versa is not true in every cases.(14,15,16)

The mandibular first molars usually have two roots, one mesial and one distal, with usual root canal distribution as two canals in the mesial root and one or two canals in the distal root.(1) The presence of a middle mesial canal with incidence of 1-15\% is major variant of root canal system of mandibular first molar. Other common variant is three canals in the distal root with an incidence of $1.7 \%$ in the Indian population; $0.2 \%$ in Senegalese population $; 1.7 \%$ in Turkish population; $0.7 \%$ in Burmese population and 3\% in Sudanese population.(17,18)

After the clinical and radiographic diagnosis, access cavity preparation and the location of the canal orifice of the RE can be difficult. The orifice of the radix root is generally located disto- to mesio-lingually from the main canal or canals in the distal root. The overlying dentine or pulp roof remnants must have to be removed to locate the entrance or orifice of the canal. So, the modification of the classical triangular access cavity to a trapezoidal form is necessary to locate and access the root canal, avoiding gouging or excessive removal of the dentin(2,4)

The knowledge of visualizing the dentinal map and canal bleeding points, law of symmetry, law of orifice location is crucial to locate the extra canals. The tactile methods using hand instrument like endodontic explorer, DG 16 probe, pathfinders and micro-openers are also helpful to locate orifices. The champagne bubble test is another useful means of locating the orifice of the canals. Th effervescent bubbles produced by the remaining pupal tissues when sodium hypochlorite is placed in the pulp chambers also helps in localizing the canal orifice. Advanced imaging techniques such as digital radiography, fiber-optic illumination, surgical loupes, dental operating microscope and micro-computed tomography (CT) are also helpful in the detection of Radix entomolaris.(1)

In the present case series, the variations in distal root anatomy were identified through careful reading of angled IOPA radiographs. The first radiograph was taken with conventional angulation, and the second one with a mesial shift of approximately $20^{\circ}$. This buccal object rule has also been called same lingual, opposite buccal rule/Clark's rule/Walton's projection. An additional root appears as a shadow or a thin radiolucent line in the radiographs.(1)

\section{Conclusion:-}

Correct diagnosis leads to correct treatment is the saying, therefore the clinician should be aware of variations in the root canal morphology of tooth for endodontic success. A case of radix entomolaris and paramolaris may be difficult but can be easily detected by a careful evaluation of pre-operative radiographs when taken at different angulations. The careful examination of the floor of the pulp chamber reveals clues for locating any extra canals if present. With advent of modern techniques and instruments tooth with morphological variations such as RE can be successfully treated endodontically provided that these variations are known to the clinician.

Ability to correctly interpret the radiograph, careful inspection of the pulp chamber floor, and use of recent concepts in access cavity preparation along with the sound knowledge of the variable anatomy of the root canal are very important for the clinician to locate and treat the root canals in case of RE or paramolaris. 


\section{References:-}

1. Arun J, Mayuri BS, Nandini TN, MallikarjunGoud . Radix entomolaris and paramolaris: A case series.International Journal of Oral Health Sciences | Jan-Jun 2015 | Vol 5 | Issue 1.

2. PJ van der Vyver, M Vorster.RadixEntomolaris: Literature review and case report.SADJ April 2017 , Vol 72 no 3 p113 - p117

3. F. Abella1, S. Patel, F. Dura' n-Sindreu, M. Mercade'\& M. Roig. Mandibular first molars with disto-lingual roots: review and clinical management. International Endodontic Journal, 45, 963-978, 2012

4. R. J. G. De Moor, C. A. J. G. Deroose\& F. L. G. Calberson. The radix entomolaris in mandibular first molars: an endodontic challenge. International Endodontic Journal, 37, 789-799, 2004

5. AmitParashar, Shikha Gupta, AbhishekZingade and ShashiParashar. The Radix Entomolaris and Paramolaris: A Review and Case Reports with Clinical Implications. J Interdiscipl Med Dent Sci 2015, 3:1

6. Rajeev Pandey, Rajat Gupta1, NitinBhagat, AnitKhatri.Endodontic Management of a Rare Anatomic variation Radix Entomolaris of Mandibular I Molar- Case Report.International Journal of Contemporary Medical Research Volume 4 | Issue 1 | January 2017 | ICV (2015): 77.83 |

7. Carlsen O, Alexandersen V (1990) Radix entomolaris: identification and morphology. Scand J Dent Res 98: 363-373.

8. Song JS, Choi HJ, Jung IY, Jung HS, Kim SO (2010) The prevalence and morphologic classification of distolingual roots in the mandibular molars in a Korean population. J Endod 36: 653-657.

9. Gu Y1, Zhou P, Ding Y, Wang P, Ni L (2011) Root canal morphology of permanent three-rooted mandibular first molars: Part III-An odontometric analysis. J Endod 37: 485-490.

10. Monika Khangwal, Monika Khokhar, ArjuneSoni, RavinderSolanki. Endodontic Management of Radix Entomolaris: Case Report International Journal of Enhanced Research in Medicines \& Dental Care (IJERMDC), ISSN: 2349-1590, Vol. 4 Issue 6, June-2017, Impact Factor: 1.102

11. Klein RM, Blake SA, Nattress BR, Hirschmann PN (1997) Evaluation of X-ray beam angulation for successful twin canal identification in mandibular incisors. IntEndod J 30: 58-63.

12. Ingle JI, Heithersay, GS, Hatwell GR (2002) Endodontic Diagnostic Procedures. BC Decker, London, UK.

13. Carlsen O, Alexandersen V (1991) Radix paramolaris in permanent mandibular molars: identification and morphology. Scand J Dent Res 99:189-195.

14. Sperber GH, Moreau JL (1998) Study of the number of roots and canals in Senegalese first permanent mandibular molars. IntEndod J 31:117-122.

15. Carlsen O, Alexandersen V (1999) Radix paramolaris and radix distomolaris in Danish permanent maxillary molars. ActaOdontolScand 57: 283-289.

16. Brabant H, Klees, L, Werelds, RJ (1958) Anomalies, mutilations et tumeurs des dents humanies. Editions Julien Prelated. Paris, France.

17. Shweta Jain. Mandibular first molar with three distal canals. Journal of Conservative Dentistry | Oct-Dec 2011 | Vol 14 | Issue 4

18. ParulBansal, Vineeta Nikhil, ShashankShekhar. Three distal root canals in mandibular first molar with different canal configurations: Report of two cases and literature review.Saudi Endodontic Journal • Jan-Apr $2015 \bullet$ Vol $5 \cdot$ Issue 1 